\title{
SOLUTIONS OF PERIOD FOUR FOR A NON-LINEAR DIFFERENCE EQUATION
}

\author{
A. BROWN ${ }^{1}$
}

(Received 22 August 1983)

\begin{abstract}
The paper extends earlier work by using the factorisation method to discuss solutions of period four for the difference equation

$$
x_{n+1}=a x_{n}^{3}+(1-a) x_{n} \quad(0<a \leqslant 4) .
$$

This equation was suggested by R. M. May as a simple mathematical model for the effect of frequency-dependent selection in genetics. It is shown that for a given value of the parameter, $a$, the identification of solutions of period four can be reduced to finding real roots for a polynomial equation of degree eight. The appropriate values of $x_{n}$ follow from a quartic equation. By splitting up the problem in this way it becomes relatively straightforward to determine the critical values of $a$ at which the various solutions of period four first appear and to discuss the stability of these solutions. Intervals of stability are tabulated in the paper.
\end{abstract}

\section{Introduction}

The present paper is an extension of an earlier one [4] in which the motivation for the work was discussed in the first two paragraphs. This followed May [6, 7] who linked the equation

$$
x_{n+1}=F\left(x_{n}\right)=a x_{n}^{3}+(1-a) x_{n}
$$

with the phenomenon of frequency-dependent selection in population growth and suggested that it merited further investigation. He noted that the parameter $a$ had to be restricted to an interval $0<a \leqslant 4$ to agree with the genetics problem and it will be shown later that the interval $2<a \leqslant 4$ suffices for the purposes of this paper. Similarly, we can restrict $x_{n}$ to the interval $[-1,1]$.

\footnotetext{
${ }^{1}$ Department of Theoretical Physics, Research School of Physical Sciences, Australian National University, G.P.O. Box 4, Canberra, A.C.T. 2601.

(C) Copyright Australian Mathematical Society 1984, Serial-fee code 0334-2700/84
} 
In the papers cited above, May showed that solutions with period two are available for $a>2$, although these solutions are unstable for $a>1+\sqrt{5}$. Experience with the logistic difference equation suggests that solutions with period four should first appear at $a=1+\sqrt{5}$ and this is confirmed by the discussion in this paper. As $a$ increases, other families of solutions of period four can be distinguished and it is of interest to determine the critical value of $a$ and the stability interval for each family.

If we refer to a cyclic solution with minimum period four as a $\mathrm{C} 4$ solution and write $\left(b_{1}, b_{2}, b_{3}, b_{4}\right)$ for the elements of the solution, then $\left(-b_{1},-b_{2},-b_{3},-b_{4}\right)$ is also a $C 4$ solution because $F\left(-b_{1}\right)=-F\left(b_{1}\right)=-b_{2}$, and so on. The existence of these "mirror image" solutions simplifies the problem for we can assume to begin with that $\alpha \geqslant 0$, where

$$
\alpha=b_{1}+b_{2}+b_{3}+b_{4},
$$

and we can include appropriate mirror image solutions later.

Iterating equation (1.1) four times gives $x_{n+4}$ as a polynomial of degree 81 in $x_{n}$ and hence the condition $x_{n+4}=x_{n}$ produces a polynomial equation of degree 81 . We can write this equation as $G\left(x_{n}\right)=x_{n+4}-x_{n}=0$. However this condition includes solutions with minimum period two ( $\mathrm{C} 2$ solutions) and the three equilibrium solutions ( $\mathrm{C} 1$ solutions) as special cases and these special cases contribute a factor of degree 9 to $G\left(x_{n}\right)$. The remaining factor gives an equation $H\left(x_{n}\right)=0$, where $H$ is of degree 72 in $x_{n}$. Solving this equation directly would be a formidable proposition and the aim of the factorisation method is to split the problem into two simpler steps. As the factorisation method has been applied in earlier papers $[2,3,4]$, it will be summarised fairly briefly in this paper.

Section 2 introduces some of the notation that is used later and goes on to discuss a special case where a full solution is possible. Section 3 lists a number of equations which are useful in the general case and Section 4 shows how they can be combined to give the key equation for solving the problem. Section 5 gives an expression for the stability criterion which is then applied to the special case mentioned in Section 2. A result for the case where $a=4$ is also included.

In the factorisation method the value of $\alpha$ is used to identify a particular solution and the main problem is to know which values of $\alpha$ are appropriate for a given value of $a$. The key equation in Section 4 is an eighth degree polynomial whose roots provide the desired values for $\alpha$. The values of $b_{1}$ to $b_{4}$ then come from a quartic equation and Section 6 discusses the solution of this quartic. It is essential to know when it will give real solutions for the elements and in this problem there are special features which make it easier to decide. In effect, the solution of the quartic is achieved by solving two separate quadratics.

Section 7 lists the numerical results that were obtained. This includes the critical values of $a$ at which the different families of solution appear and the upper limits of $a$ for stability in the cases where stable solutions occur. 


\section{Introductory ideas and a special case}

For a $\mathrm{C} 4$ solution $\left(b_{1}, b_{2}, b_{3}, b_{4}\right)$, the basic equation is that

$$
b_{i+1}=F\left(b_{i}\right)=a b_{i}^{3}+(1-a) b_{i},
$$

for $i=1,2,3,4$, with $b_{5}=b_{1}$. This C4 solution contributes a factor $h(x)$ to $H(x)$, where

$$
\begin{aligned}
h(x) & =\left(x-b_{1}\right)\left(x-b_{2}\right)\left(x-b_{3}\right)\left(x-b_{4}\right) \\
& =x^{4}-\alpha x^{3}+\beta x^{2}-\gamma x+\delta,
\end{aligned}
$$

an equation which defines $\alpha, \beta, \gamma, \delta$ as symmetrical functions of the $b$ 's. At most there can be 18 factors of this type in $H(x)$ and some of them will be related because they correspond to mirror image solutions. If there is a distinct mirror image solution $\left(-b_{1},-b_{2},-b_{3},-b_{4}\right)$ its contribution to $H(x)$ is a factor

$$
\begin{aligned}
h^{*}(x) & =\left(x+b_{1}\right)\left(x+b_{2}\right)\left(x+b_{3}\right)\left(x+b_{4}\right) \\
& =x^{4}+\alpha x^{3}+\beta x^{2}+\gamma x+\delta
\end{aligned}
$$

and the factors $h(x)$ and $h^{*}(x)$ combine to give

$$
\begin{aligned}
k(x) & =h(x) h^{*}(x)=\left(x^{2}-b_{1}^{2}\right)\left(x^{2}-b_{2}^{2}\right)\left(x^{2}-b_{3}^{2}\right)\left(x^{2}-b_{4}^{2}\right) \\
& =x^{8}-A x^{6}+B x^{4}-C x^{2}+D,
\end{aligned}
$$

where

$$
A=\alpha^{2}-2 \beta, \quad B=\beta^{2}-2 \alpha \gamma+2 \delta, \quad C=\gamma^{2}-2 \beta \delta, \quad D=\delta^{2} .
$$

It turns out that at most $H(x)$ provides eight factors of type $k(x)$, where the mirror image solutions are distinct, and two factors of type $h(x)$ where the mirror image solution is the same as the original solution.

When we say that the mirror image solution is not distinct we mean that $\left(-b_{1},-b_{2},-b_{3},-b_{4}\right)$ gives the same $C 4$ solution as $\left(b_{1}, b_{2}, b_{3}, b_{4}\right)$, whether or not the elements are ordered in the same way. This means that we have the same solution if

$$
-b_{1}=b_{1} \text { or }-b_{2}=b_{1} \text { or }-b_{3}=b_{1} \text { or }-b_{4}=b_{1} \text {. }
$$

The first of these conditions gives the equilibrium solution $b_{1}=0$, which we can ignore, and the second gives

$$
b_{3}=F\left(b_{2}\right)=-F\left(b_{1}\right)=-b_{2}=b_{1} \text {. }
$$

It follows that $b_{4}=F\left(b_{3}\right)=F\left(b_{1}\right)=b_{2}$ and thus we have a C2 solution. In the same way, $b_{1}=-b_{4}$ leads to a $\mathrm{C} 2$ solution, so we can ignore both of these cases. This leaves only the case $b_{1}=-b_{3}$ and when this holds

$$
b_{4}=F\left(b_{3}\right)=-F\left(b_{1}\right)=-b_{2},
$$

so we have simultaneously

$$
b_{1}+b_{3}=0, \quad b_{2}+b_{4}=0 .
$$


It follows that $h(x)=\left(x-b_{1}\right)\left(x-b_{2}\right)\left(x+b_{1}\right)\left(x+b_{2}\right)$ and hence

$$
\alpha=0, \quad \beta=-b_{1}^{2}-b_{2}^{2}, \quad \gamma=0, \quad \delta=b_{1}^{2} b_{2}^{2},
$$

while the basic equations reduce to

$$
\begin{gathered}
b_{2}=b_{1}\left\{a b_{1}^{2}+(1-a)\right\}, \\
b_{3}=-b_{1}=b_{2}\left\{a b_{2}^{2}+(1-a)\right\} .
\end{gathered}
$$

Multiplying together these equations gives

$$
-b_{1} b_{2}=b_{1} b_{2}\left\{a^{2} \delta-a(1-a) \beta+(1-a)^{2}\right\} .
$$

If we assume $b_{1} b_{2} \neq 0$, to avoid an equilibrium solution, we get

$$
-1=a^{2} \delta-a(1-a) \beta+(1-a)^{2} .
$$

Also, if we multiply equation (2.9) by $b_{1}$ and equation (2.10) by $b_{2}$, then add, the result is

$$
0=a\left(b_{1}^{4}+b_{2}^{4}\right)+(1-a)\left(b_{1}^{2}+b_{2}^{2}\right)=a\left(\beta^{2}-2 \delta\right)-(1-a) \beta .
$$

Eliminating $\delta$ gives

$$
0=a^{2} \beta^{2}-3 a(1-a) \beta+\left(2 a^{2}-4 a+4\right)
$$

and hence

$$
2 a \beta=3(1-a) \pm \sqrt{\left(a^{2}-2 a-7\right)} .
$$

This means that the solution for $\beta$ is not real unless $a^{2}-2 a-7 \geqslant 0$, that is unless $a \geqslant 1+2 \sqrt{2}$. Thus the critical value for this type of solution is $a^{*}=1+$ $2 \sqrt{2}$. When $a=a^{*}$, there is a single solution, with $\beta=-(3 \sqrt{2}) / a^{*}, \delta=3 /\left(a^{*}\right)^{2}$, $b_{1}^{2}=(3+\sqrt{3}) /\left(a^{*} \sqrt{2}\right), b_{2}^{2}=(3-\sqrt{3}) /\left(a^{*} \sqrt{2}\right)$.

For $a>a^{*}$, there are two solutions, one with

$$
2 a \beta=3(1-a)+Q, \quad 2 a^{2} \delta=\left(a^{2}-2 a-1\right)+(1-a) Q,
$$

where $Q=\sqrt{\left(a^{2}-2 a-7\right)}$, and the other with $+Q$ replaced by $-Q$ in equations (2.15). It can be checked that these relationships lead to real values for $b_{1}$ and $b_{2}$. The stability of these solutions is discussed later, in Section 5.

It is useful to note that these are the only C4 solutions for which $\alpha=0$. If we add the four equations (2.1) we get

$$
\alpha=\sum b_{i+1}=a \sum b_{i}^{3}+(1-a) \sum b_{i}=a \sum b_{i}^{3}+(1-a) \alpha,
$$

where the summation is over $i=1$ to $i=4$. Taking $a>0$, we get

$$
\alpha=\sum b_{i}^{3}=\alpha^{3}-3 \alpha \beta+3 \gamma
$$

and hence

$$
\gamma=(\alpha / 3)\left(1-\alpha^{2}+3 \beta\right) \text {. }
$$


This means that if $\alpha=0$, then $\gamma$ is also zero. Now $\alpha=0$ implies that $b_{1}+b_{3}=$ $-\left(b_{2}+b_{4}\right)$ and from this

$$
\gamma=b_{1} b_{3}\left(b_{2}+b_{4}\right)+b_{2} b_{4}\left(b_{1}+b_{3}\right)=\left(b_{1} b_{3}-b_{2} b_{4}\right)\left(b_{2}+b_{4}\right) \text {. }
$$

Since $\gamma=0$, we have $b_{1} b_{3}=b_{2} b_{4}$ or $b_{2}+b_{4}=0$. If $b_{2}+b_{4}=0$, we are back to equation (2.7) and the $C 4$ solutions already discussed. If $b_{1} b_{3}=b_{2} b_{4}$, together with $b_{1}+b_{3}=-\left(b_{2}+b_{4}\right)$, then we must have either $b_{1}=-b_{2}, b_{3}=-b_{4}$ or $b_{1}=-b_{4}, b_{3}=-b_{2}$. Either case leads to a $\mathrm{C} 2$ or $\mathrm{C} 1$ solution. Thus the only $\mathrm{C} 4$ solutions which have $\alpha=0$ are the special cases considered above.

\section{Additional relationship in the general case}

The first step in the factorisation method is to obtain equations which allow $\beta$, $\gamma$ and $\delta$ to be evaluated for given values of $a$ and $\alpha$. The basic equations available are equations (2.1) and in obtaining equation (2.16) we have already had an example of how they can be used. In addition we need an equation which allows us to find suitable values of $\alpha$ when $a$ is specified. It turns out that this is a polynomial equation of degree 8 in $\alpha^{2}$ and, for a given $a$, we have to determine the positive real roots of this equation. Each root gives a value of $\alpha$, which we can take as positive, and from this we get corresponding values of $\beta, \gamma, \delta$. Finally, the $b_{i}$ can be determined as the roots of

$$
h(x)=x^{4}-\alpha x^{3}+\beta x^{2}-\gamma x+\delta=0 .
$$

The laborious part is setting up the required relationships and the equation for $\alpha$. Once this has been done the computational work is straightforward.

Because equations (2.1) have cyclic symmetry in the elements $b_{i}$, rather than full permutational symmetry, it is convenient to subdivide $\beta$ into two parts, each with cyclic symmetry, and also to use $\Sigma$ as a cyclic summation symbol. We can write

$$
\begin{aligned}
& \beta_{1}=\sum b_{1} b_{2}=b_{1} b_{2}+b_{2} b_{3}+b_{4} b_{1}, \\
& \beta_{2}=b_{1} b_{3}+b_{2} b_{4}=(1 / 2) \sum b_{1} b_{3},
\end{aligned}
$$

with $\beta=\beta_{1}+\beta_{2}$. Purely algebraic expansions, without using equations (2.1), give a number of relationships such as

$$
\begin{gathered}
\alpha \gamma-4 \delta-\beta_{1} \beta_{2}=\sum b_{1} b_{2}^{2} b_{3}, \\
\beta^{2}-2 \alpha \gamma+4 \delta-\beta_{2}^{2}=\sum b_{1}^{2} b_{2}^{2}, \\
A \beta_{2}=\beta_{2} \sum b_{1}^{2}=\sum\left(b_{1}^{3} b_{3}+b_{1} b_{2}^{2} b_{3}\right), \\
A \beta_{1}-\beta_{1} \beta_{2}=\sum\left\{\left(b_{2}+b_{4}\right) b_{1}^{3}\right\}, \\
\beta_{1}\left(\beta_{2}^{2}-2 \delta\right)=\sum\left(b_{1}^{3} b_{2} b_{3}^{2}+b_{1}^{2} b_{2} b_{3}^{3}\right),
\end{gathered}
$$


as well as the more familiar relationships

$$
\begin{aligned}
& \sum b_{1}^{2}=A=\alpha^{2}-2 \beta, \\
& \sum b_{1}^{4}=A^{2}-2 B=\alpha^{4}-4 \alpha^{2} \beta+2 \beta^{2}+4 \alpha \gamma-4 \delta, \\
& \sum b_{1}^{6}=A^{3}-3 A B+3 C .
\end{aligned}
$$

If we take $b_{2}=a b_{1}^{3}+(1-a) b_{1}$ as our paradigm for equation (2.1) we can obtain the relationships

$$
\begin{aligned}
\beta_{1} & =\sum b_{1} b_{2}=a \sum b_{1}^{4}+(1-a) \sum b_{1}^{2}=a\left(A^{2}-2 B\right)+(1-a) A, \\
A & =\sum b_{2}^{2}=a \sum b_{1}^{3} b_{2}+(1-a) \beta_{1}, \\
\beta_{1} & =\sum b_{2} b_{3}=a \sum b_{1}^{3} b_{3}+2(1-a) \beta_{2}, \\
2 \beta_{2} & =\sum b_{2} b_{4}=a \sum b_{1}^{3} b_{4}+(1-a) \beta_{1}, \\
a^{2} \sum b_{1}^{6} & =\sum\left\{b_{2}-(1-a) b_{1}\right\}^{2}=A\left(a^{2}-2 a+2\right)-2(1-a) \beta_{1} .
\end{aligned}
$$

If we multiply all four of the basic equations together, we get

$$
b_{2} b_{3} b_{4} b_{1}=\left(b_{1} b_{2} b_{3} b_{4}\right) \prod\left\{a b_{1}^{2}+(1-a)\right\},
$$

where $\Pi$ is used-as a cyclic product symbol. We can take $b_{1} b_{2} b_{3} b_{4}$ as a nonzero factor (to avoid the equilibrium solution $b_{i}=0$ ) and expand the product on the right-hand side. This gives

$$
1=a^{4} D+a^{3}(1-a) C+a^{2}(1-a)^{2} B+a(1-a)^{3} A+(1-a)^{4} .
$$

Similarly, we can write

$$
\begin{aligned}
& b_{4}-b_{2}=\left(b_{3}-b_{1}\right)\left\{a\left(b_{1}^{2}+b_{1} b_{3}+b_{3}^{2}\right)+(1-a)\right\}, \\
& b_{3}-b_{1}=\left(b_{2}-b_{4}\right)\left\{a\left(b_{2}^{2}+b_{2} b_{4}+b_{4}^{2}\right)+(1-a)\right\} .
\end{aligned}
$$

and take $b_{4} \neq b_{2}$ and $b_{3} \neq b_{1}$ to avoid a $C 2$ solution. Multiplying the equations together and cancelling a nonzero factor $\left(b_{4}-b_{2}\right)\left(b_{3}-b_{1}\right)$ leaves

$$
\begin{aligned}
-1 & =\left\{a\left(b_{1}^{2}+b_{1} b_{3}+b_{3}^{2}\right)+(1-a)\right\}\left\{a\left(b_{2}^{2}+b_{2} b_{4}+b_{4}^{2}\right)+(1-a)\right\} \\
& =a^{2}\left(\delta+\sum b_{1}^{2} b_{2}^{2}+\sum b_{1} b_{2}^{2} b_{3}\right)+a(1-a)\left(A+\beta_{2}\right)+(1-a)^{2} .
\end{aligned}
$$

In the same way,

$$
\begin{aligned}
& b_{4}+b_{2}=\left(b_{3}+b_{1}\right)\left\{a\left(b_{1}^{2}-b_{1} b_{3}+b_{3}^{2}\right)+(1-a)\right\}, \\
& b_{1}+b_{3}=\left(b_{2}+b_{4}\right)\left\{a\left(b_{2}^{2}-b_{2} b_{4}+b_{4}^{2}\right)+(1-a)\right\},
\end{aligned}
$$


and assuming $\left(b_{1}+b_{3}\right)\left(b_{2}+b_{4}\right) \neq 0$ leads to

$$
1=a^{2}\left(\delta+\sum b_{1}^{2} b_{2}^{2}-\sum b_{1} b_{2}^{2} b_{3}\right)+a(1-a)\left(A-\beta_{2}\right)+(1-a)^{2} .
$$

By adding equations (3.18) and (3.21)

$$
0=a^{2}\left(\delta+\sum b_{1}^{2} b_{2}^{2}\right)+a(1-a) A+(1-a)^{2},
$$

and it follows that

$$
-1=a^{2} \sum b_{1} b_{2}^{2} b_{3}+a(1-a) \beta_{2} .
$$

Other equations are available but we have enough for the moment.

\section{Combining the various relationships}

From equation (2.16)

$$
3 \alpha \gamma=\alpha^{2}\left(1-\alpha^{2}+3 \beta\right)
$$

and we can combine this with equations (3.4) and (3.23) to give

$$
12 a^{2} \delta=a^{2} \alpha^{2}\left(1-\alpha^{2}+3 \beta\right)-3 a^{2} \beta_{1} \beta_{2}+3+3 a(1-a) \beta_{2} .
$$

From equations (3.5) and (3.22),

$$
a^{2}(2 \alpha \gamma-5 \delta)=a^{2}\left(\beta_{1}^{2}+2 \beta_{1} \beta_{2}\right)+a(1-a) A+(1-a)^{2}
$$

and replacing $\alpha \gamma$ and $\delta$ from equations (4.1) and (4.2) leads to

$$
\begin{aligned}
a^{2}\left(4 \beta_{1}^{2}+3 \beta_{1} \beta_{2}\right)= & -\left(4 a^{2}-8 a+9\right)+\left(5 a^{2}-4 a\right) \alpha^{2}-a^{2} \alpha^{4} \\
& +\beta_{1}\left(8 a-8 a^{2}+3 a^{2} \alpha^{2}\right)+\beta_{2}\left(3 a-3 a^{2}+3 a^{2} \alpha^{2}\right) .
\end{aligned}
$$

We can obtain a second relationship between $\beta_{1}$ and $\beta_{2}$ by using equations (3.14) and (3.23) in equation (3.6). This gives

$$
2 a^{2}\left(\beta_{1} \beta_{2}+\beta_{2}^{2}\right)=1-a \beta_{1}+\left(3 a-3 a^{2}+a^{2} \alpha^{2}\right) \beta_{2} .
$$

In the same way, combining equations (3.7), (3.13) and (3.15) leads to

$$
a^{2}\left(2 \beta_{1}^{2}+3 \beta_{1} \beta_{2}\right)=-a \alpha^{2}+\left(4 a-2 a^{2}+a^{2} \alpha^{2}\right) \beta_{1} .
$$

Equations (4.4), (4.5) and (4.6) provide three relationships between $\beta_{1}$ and $\beta_{2}$ so it should be possible to eliminate $\beta_{1}$ and $\beta_{2}$ and obtain an equation linking $a$ with $\alpha$. From the theory of resultants [1], this would lead most directly to a $7 \times 7$ determinant, although a smaller determinant $(4 \times 4)$ would be possible [5]. It 
would be necessary also to solve for $\beta_{1}$ and $\beta_{2}$ from these quadratic forms. Instead of pursuing this approach the available equations were manipulated to give three linear relationships between $\beta_{1}$ and $\beta_{2}$, which made it easy to solve for $\beta_{1}$ and $\beta_{2}$ and to write down the equation for $\alpha$ as a $3 \times 3$ determinant.

The first step was to solve for $\beta_{1}^{2}, \beta_{1} \beta_{2}$ and $\beta_{2}^{2}$ as linear functions of $\beta_{1}$ and $\beta_{2}$. This gave

$$
\begin{aligned}
2 a^{2} \beta_{1}^{2}= & -a^{2} \alpha^{4}+\left(5 a^{2}-3 a\right) \alpha^{2}-\left(4 a^{2}-8 a+9\right) \\
& +\left(4 a-6 a^{2}+2 a^{2} \alpha^{2}\right) \beta_{1}+\left(3 a-3 a^{2}+3 a^{2} \alpha^{2}\right) \beta_{2}, \\
3 a^{2} \beta_{1} \beta_{2}= & a^{2} \alpha^{4}+\left(2 a-5 a^{2}\right) \alpha^{2}+\left(4 a^{2}-8 a+9\right) \\
& +\left(4 a^{2}-a^{2} \alpha^{2}\right) \beta_{1}+\left(3 a^{2}-3 a-3 a^{2} \alpha^{2}\right) \beta_{2}, \\
6 a^{2} \beta_{2}^{2}= & -2 a^{2} \alpha^{4}+\left(10 a^{2}-4 a\right) \alpha^{2}-\left(8 a^{2}-16 a+15\right) \\
& +\left(2 a^{2} \alpha^{2}-3 a-8 a^{2}\right) \beta_{1}+\left(15 a-15 a^{2}+9 a^{2} \alpha^{2}\right) \beta_{2} .
\end{aligned}
$$

By using these equations it was straightforward to express $\beta^{2}, \delta, B, C, D, A^{2}, A B$ and $A^{3}$ as linear functions of $\beta_{1}$ and $\beta_{2}$ and thus make use of equations (3.11), (3.12), (3.16) and (3.17). Equation (3.12) did not give any additional information, although it served as a check. Equations (3.11) and (3.16) gave the linear equation

$$
\beta_{1} P_{1}+\beta_{2} Q_{1}=R_{1} \text {, }
$$

where

$$
\begin{aligned}
P_{1}= & 4 a^{3} \alpha^{4}+\left(9 a^{2}-20 a^{3}\right) \alpha^{2}+\left(16 a^{3}-45 a^{2}+9 a\right), \\
Q_{1}= & 15 a^{3} \alpha^{4}+\left(45 a^{2}-30 a^{3}\right) \alpha^{2}+\left(15 a^{3}-45 a^{2}\right), \\
R_{1}= & 4 a^{3} \alpha^{6}+\left(17 a^{2}-24 a^{3}\right) \alpha^{4} \\
& +\left(36 a^{3}-85 a^{2}+54 a\right) \alpha^{2}+\left(72-99 a+68 a^{2}-16 a^{3}\right) .
\end{aligned}
$$

A second linear equation came from equation (3.8), where $b_{1}^{3}$ and $b_{3}^{3}$ can be replaced by $(1 / a)\left\{b_{2}-(1-a) b_{1}\right\}$ and $(1 / a)\left\{b_{4}-(1-a) b_{3}\right\}$, respectively. This leads to the equation

$$
a \beta_{1}\left(\beta_{2}^{2}-2 \delta\right)=\beta_{1}^{2}-\alpha \gamma+a \beta_{1} \beta_{2} .
$$

Some straightforward algebra, using equations (4.7) to (4.9), produced an equation

$$
\beta_{1} P_{2}^{*}+\beta_{2} Q_{2}^{*}=R_{2}^{*}
$$

where

$$
\begin{aligned}
& P_{2}^{*}=6 a^{3} \alpha^{4}+\left(24 a^{2}-30 a^{3}\right) \alpha^{2}+\left(24 a^{3}-78 a^{2}+54 a\right), \\
& Q_{2}^{*}=12 a^{3} \alpha^{4}+\left(45 a^{2}-30 a^{3}\right) \alpha^{2}+\left(18 a^{3}-63 a^{2}+45 a\right),
\end{aligned}
$$




$$
\begin{aligned}
R_{2}^{*}= & 4 a^{3} \alpha^{6}+\left(21 a^{2}-26 a^{3}\right) \alpha^{4} \\
& +\left(46 a^{3}-111 a^{2}+75 a\right) \alpha^{2}-\left(24 a^{3}-108 a^{2}+174 a-135\right)
\end{aligned}
$$

In the later work, equation (4.15) was replaced by

$$
\beta_{1} P_{2}+\beta_{2} Q_{2}=R_{2} \text {, }
$$

with

$$
P_{2}=P_{1}-P_{2}^{*}, \quad Q_{2}=Q_{1}-Q_{2}^{*}, \quad R_{2}=R_{1}-R_{2}^{*},
$$

since this gave slightly simpler terms in the linear equation.

Equation (3.17) also leads to a linear equation in $\beta_{1}$ and $\beta_{2}$ but it turned out that this was a linear combination of equations (4.10) and (4.19). It served as a check on the coefficients in these equations and in the numerical calculations equation (3.17) was used as a check on the values obtained for $A, B, C$ and $D$.

A third linear equation was obtained by combining equations (4.8), (4.9) and (4.19). From equations (4.8) and (4.9), we can write

$$
6 a^{2} \beta_{1} \beta_{2}=S_{1}+T_{1} \beta_{1}+V_{1} \beta_{2}, \quad 6 a^{2} \beta_{2}^{2}=S_{2}+T_{2} \beta_{1}+V_{2} \beta_{2},
$$

where $S_{1}, T_{1}, V_{1}, S_{2}, T_{2}$ and $V_{2}$ are functions of $a$ and $\alpha^{2}$, and from this

$$
\begin{aligned}
6 a^{2} R_{2} \beta_{2} & =6 a^{2}\left(P_{2} \beta_{1}+Q_{2} \beta_{2}\right) \beta_{2} \\
& =P_{2}\left(S_{1}+T_{1} \beta_{1}+V_{1} \beta_{2}\right)+Q_{2}\left(S_{2}+T_{2} \beta_{1}+V_{2} \beta_{2}\right) .
\end{aligned}
$$

This gives a linear equation

$$
\beta_{1} P_{3}^{*}+\beta_{2} Q_{3}^{*}=R_{3}^{*}
$$

with

$$
P_{3}^{*}=P_{2} T_{1}+Q_{2} T_{2}, \quad Q_{3}^{*}=P_{2} V_{1}+Q_{2} V_{2}-6 a^{2} R_{2}, \quad R_{3}^{*}=-P_{2} S_{1}-Q_{2} S_{2}
$$

In practice, equation (4.21) was replaced by

$$
\beta_{1} P_{3}+\beta_{2} Q_{3}=R_{3},
$$

where

$$
P_{3}=(2 / a) P_{3}^{*}+\left(5 a-3-5 a \alpha^{2}\right) P_{1},
$$

with similar definitions for $Q_{3}$ and $R_{3}$. In more detail,

$$
\begin{aligned}
P_{3}= & -15 a^{3} \alpha^{4}+\left(30 a^{3}-72 a^{2}\right) \alpha^{2}+\left(-15 a^{3}+72 a^{2}+243 a\right), \\
Q_{3}= & 3 a^{4} \alpha^{6}+\left(72 a^{3}-33 a^{4}\right) \alpha^{4} \\
& +\left(57 a^{4}-144 a^{3}+27 a^{2}\right) \alpha^{2}-27 a^{4}+72 a^{3}+189 a^{2}-54 a,
\end{aligned}
$$




$$
\begin{aligned}
R_{3}= & 3 a^{3} \alpha^{6}-\left(18 a^{3}+39 a^{2}\right) \alpha^{4} \\
& +\left(27 a^{3}+60 a^{2}+18 a\right) \alpha^{2}-12 a^{3}-21 a^{2}+9 a+54 .
\end{aligned}
$$

Equations (4.10), (4.19) and (4.22) are linearly independent and the determinant

$$
\Delta=\left|\begin{array}{lll}
P_{1} & Q_{1} & R_{1} \\
P_{2} & Q_{2} & R_{2} \\
P_{3} & Q_{3} & R_{3}
\end{array}\right|
$$

is a polynomial of degree 8 in $\alpha^{2}$. If we put $Y=a \alpha^{2}$, the equation which determines $\alpha^{2}$ for a given valule of $a$ can be written as $M(Y, a)=0$, where

$$
M(Y, a)=\sum_{n=0}^{8} c_{n} Y^{8-n},
$$

with

$$
\begin{aligned}
c_{0}= & 1, \quad c_{1}=21(1-a), \quad c_{2}=216-330 a+162 a^{2}, \\
c_{3}= & 1359-2403 a+1902 a^{2}-602 a^{3}, \\
c_{4}= & 4617-10728 a+9558 a^{2}-5160 a^{3}+1173 a^{4}, \\
c_{5}= & 10611-25839 a+27108 a^{2}-16740 a^{3}+7053 a^{4}-1233 a^{5}, \\
c_{6}= & 18144-32724 a+37260 a^{2}-26496 a^{3}+13662 a^{4}-4710 a^{5}+664 a^{6}, \\
c_{7}= & 7290-7614 a+14418 a^{2}-18630 a^{3} \\
& +11673 a^{4}-4617 a^{5}+1224 a^{6}-144 a^{7}, \\
c_{8}= & -37908+48114 a-21465 a^{2}-4698 a^{3} \\
& +8424 a^{4}-2916 a^{5}+324 a^{6} .
\end{aligned}
$$

For a given value of $a$, the coefficients $c_{1}$ to $c_{8}$ can be evaluated and we then want the positive real roots of $M(Y, a)=0$. For each of these roots we get a corresponding value of $\alpha$, which we can take as positive. Then $\beta_{1}$ and $\beta_{2}$ can be determined from equations (4.10) and (4.19) and $\beta$ is simply $\beta_{1}+\beta_{2}$. Equation (2.16) gives $\gamma$ and $\delta$ could be obtained from equation (4.2), although in practice the equation used was

$$
\begin{aligned}
6 a^{2} \delta= & -a^{2} \alpha^{4}+\left(3 a^{2}-a\right) \alpha^{2}-\left(2 a^{2}-4 a+3\right) \\
& +2 a^{2}\left(\alpha^{2}-1\right) \beta_{1}+\left(3 a-3 a^{2}+3 a^{2}\right) \beta_{2},
\end{aligned}
$$

which arises from combining equations (4.2) and (4.8). $A, B, C$ and $D$ can then be evaluated from equation (2.5). 


\section{The stability criterion}

Apart from the check provided by equation (3.17), $A, B, C$ and $D$ are useful in testing the local stability of a $C 4$ solution. For local stability we must have $|S|<1$, where

$$
\begin{aligned}
S & =\prod\left\{F^{\prime}\left(b_{1}\right)\right\}=\prod\left\{3 a b_{1}^{2}+(1-a)\right\} \\
& =81 a^{4} D+27 a^{3}(1-a) C+9 a^{2}(1-a)^{2} B+3 a(1-a)^{3} A+(1-a)^{4} .
\end{aligned}
$$

In the special case considered in Section 2, both $\alpha$ and $\gamma$ are zero, so $A=-2 \beta$, $B=\beta^{2}+2 \delta, C=-2 \beta \delta, D=\delta^{2}$. In one family of solutions

$$
\begin{aligned}
2 a \beta & =3(1-a)+Q=Q-3 c, \\
2 a^{2} \delta & =a^{2}-2 a-1+(1-a) Q=c^{2}-2-c Q,
\end{aligned}
$$

where $c=a-1 \geqslant 2 \sqrt{2}$ and $Q=\sqrt{\left(a^{2}-2 a-7\right)}=\sqrt{\left(c^{2}-8\right)}$. When these values are substituted into $S$ the result is

$$
S=10 c^{4}-90 c^{2}+81+\left(54 c-6 c^{3}\right) Q .
$$

As $a \rightarrow 1+2 \sqrt{2}$ from above, $c \rightarrow 2 \sqrt{2}, Q \rightarrow 0$ and $S \rightarrow 640-720+81=1$. Thus we have a familiar situation, that the critical value of $a$ corresponds to a cycle which is at the upper limit for stability. If we form the derivative $d S / d c$, the dominant term as $a \rightarrow 1+2 \sqrt{2}$ from above is $6 c^{2}\left(9-c^{2}\right) / Q$ and this tends to $+\infty$ as $Q \rightarrow 0$. For $a=4$, we have $c=3, Q=1, S=81$ and $d S / d c=432$, so there is a strong presumption that this family of solutions is unstable, with $S>1$ for $a>a^{*}=1+2 \sqrt{2}$. (This can be shown more rigorously, as indicated below.)

In the other family of solutions, we have to replace $Q$ by $-Q$ in equations (5.2), (5.3) and (5.4). With this change, the dominant term in $d S / d c$ also shows a change in sign and tends to $-\infty$ as $c \rightarrow 2 \sqrt{2}$ from above. On the other hand, for $a=4$ we again have $S=81$ and $d S / d c$ is now 648 , which suggests that $S$ decreases to a minimum value between $c=2 \sqrt{2}$ and $c=3$ and then increases rapidly after the minimum. Numerical evidence confirms this and suggests that the minimum is zero (as happened with one family of $\mathrm{C} 2$ solutions). To check on this, put $c=(2 \sqrt{2}) \cosh u$ and take $u=0$ as corresponding to $c=2 \sqrt{2}$, with $u=u_{0}$ corresponding to $c=3$. Then $\cosh u_{0}=3 /(2 \sqrt{2})$, $\sinh u_{0}=1 /(2 \sqrt{2})$ and $\exp u_{0}=\sqrt{2}$, with $\cosh 2 u_{0}=5 / 4$ and $\sinh 2 u_{0}=3 / 4$. Note that $Q=(2 \sqrt{2}) \sinh u$. Our expression for $S$ in this case is

$$
S=10 c^{4}-90 c^{2}+81-\left(54 c-6 c^{3}\right) Q,
$$

and in terms of $u$ this becomes

$$
\begin{aligned}
S & =-39-120 \sinh 2 u-40 \cosh 2 u+48 \sinh 4 u+80 \cosh 4 u \\
& =-39-(80 \sqrt{2}) \sinh \left(2 u+u_{0}\right)+64 \cosh \left(4 u+2 u_{0}\right) .
\end{aligned}
$$


Hence

$$
d S / d u=32 \cosh \left(2 u+u_{0}\right)\left\{16 \sinh \left(2 u+u_{0}\right)-5 \sqrt{2}\right\} .
$$

It is easy to verify that $d S / d u=-48$ at $u=0$ and $d S / d u=+648$ at $u=u_{0}$, with a single zero between 0 and $u_{0}$. This corresponds to a minimum value of $S$ and at this minimum

$$
\sinh \left(2 u+u_{0}\right)=(5 \sqrt{2}) /(16), \quad \cosh \left(4 u+2 u_{0}\right)=89 / 64,
$$

which gives $S=0$ at the minimum. The minimum occurs when $c^{2}=(27+$ $9 \sqrt{17}) / 8$, which corresponds to $a=3.8308$.

For this family of solutions there is a small range of values of $a$ for which the $\mathrm{C} 4$ solutions are stable. The upper limit occurs when $S$ is again equal to 1 , after passing through the minimum. If we replace $2 u+u_{0}$ by $U$ in equation (5.6) we get $S=1$ when

$$
0=-40-(80 \sqrt{2}) \sinh U+64\left(1+2 \sinh ^{2} U\right),
$$

an equation whose solutions are

$$
\sinh U=1 /(2 \sqrt{2}) \text { and } \sinh U=(3 \sqrt{2}) / 8 .
$$

The first of these corresponds to $u=0$ and the second gives $c^{2}=\{13+$ $3 \sqrt{(41)}\} / 4$. If we use $a^{* *}$ for the corresponding value of $a$, the stability interval is $a^{*}<a<a^{* *}$, with $a^{*}=3.828427, a^{* *}=3.837665$.

When $\beta$ is given by equation (5.2) and $S$ by equation (5.4), we can use the same substitution, $c=(2 \sqrt{2}) \cosh u$, to discuss the change in $S$ as $u$ increases from 0 to $u_{0}$. In this case

$$
\begin{aligned}
S & =-39+(80 \sqrt{2}) \sinh \left(2 u-u_{0}\right)+64 \cosh \left(4 u-2 u_{0}\right) \\
d S / d u & =32 \cosh \left(2 u-u_{0}\right)\left\{16 \sinh \left(2 u-u_{0}\right)+5 \sqrt{2}\right\}
\end{aligned}
$$

which gives $d S / d u>0$ for $0 \leqslant u \leqslant u_{0}$.

Another result which arises from equation (5.1) is that $S= \pm 81$ for $a=4$. It was noted previously [4] that for $a=4$, equation (1.1) has a general solution $x_{n}=\cos \left(3^{n} \phi\right)$, where $x_{0}=\cos \phi$, and this leads to cyclic solutions for suitable values of $\phi$. In particular, $x_{n+4}=x_{n}$ when

$$
\text { (i) } \phi=N \pi / 41 \text {, or (ii) } \phi=N \pi / 40 \text {, }
$$

for any integer $N$. As it stands, this includes the $\mathrm{C} 1$ and $\mathrm{C} 2$ solutions as special cases but we can pick out the independent $\mathrm{C} 4$ solutions by choosing suitable values of $N$. For $\phi=N \pi / 41$, there are five independent solutions with $\alpha>0$ and they can be obtained by taking $N=1,2,4,7$ and 8 . Each of these solutions has a 
mirror image solution, making ten distinct solutions in all. For $\phi=N \pi / 40$, there are three independent solutions with $\alpha>0$, obtained by taking $N=1,2$ and 7 . Again, each of these has a mirror image solution. The $\mathrm{C} 4$ solutions which have $\alpha=0$ correspond to $\phi=4 \pi / 40$ and $\phi=5 \pi / 40$. Thus there are in all eighteen of these trigonometric $\mathrm{C} 4$ solutions for $a=4$, as foreshadowed in the earlier discussion.

These trigonometric solutions were invaluable for testing the equations in Sections 3 and 4 and for testing computer programmes in the numerical work.

If we take $a=4$ and $b_{1}=\cos \phi$, then

$$
3 a b_{1}^{2}+(1-a)=12 \cos ^{2} \phi-3=3(\sin 3 \phi) /(\sin \phi) .
$$

(Alternatively, equation (5.12) can be obtained by using

$$
\left.F^{\prime}\left(b_{1}\right)=d b_{2} / d b_{1}=\left(d b_{2} / d \phi\right) /\left(d b_{1} / d \phi\right) .\right)
$$

In the same way

$$
3 a b_{2}^{2}+(1-a)=3(\sin 9 \phi) /(\sin 3 \phi),
$$

and so on, with the result that

$$
S=81(\sin 81 \phi) /(\sin \phi) .
$$

For $\phi=N \pi / 40,81 \phi=2 N \pi+\phi$ and $\sin 81 \phi=\sin \phi$, so $S=+81$ for $\phi=$ $N \pi / 40$. A similar argument gives $81 \phi=2 N \pi-\phi$ and $S=-81$ when $\phi=N \pi / 41$. These results help to identify which families of solutions should have stability intervals.

A result which does not arise from equation (5.1) but which relates to stability is that for $0<a \leqslant 2$ and $\left|x_{0}\right|<1$ all solutions of equation (1.1) converge to zero as $n \rightarrow \infty$. For $0<a \leqslant 1$ this global property is fairly obvious, since convergence is from one side only. For $1<a \leqslant 2, x_{n}$ and $x_{n+1}$ can have opposite signs but for $0<\left|x_{n}\right|<1$

$$
r_{n}=x_{n+1} / x_{n}=a x_{n}^{2}+(1-a)=(1+c) x_{n}^{2}-c .
$$

As before, $c=a-1$ and the restriction on $a$ gives $0<c \leqslant 1$. It follows that

$$
\begin{gathered}
1-r_{n}=(1+c)\left(1+x_{n}^{2}\right)>0, \\
1+r_{n}=(1+c) x_{n}^{2}+(1-c)>0 .
\end{gathered}
$$

Thus $\left|r_{n}\right|<1$ and this ensures that $\left|x_{n+1}\right|<\left|x_{n}\right|$. Hence if $0<\left|x_{0}\right|<1$ the values of $x_{n}$ either move closer to zero at each step or jump to zero and stay there. Because of this global convergence property for $0<a \leqslant 2$, any $\mathrm{C} 4$ solutions must occur for $a>2$ and the numerical work was restricted accordingly. 


\section{Solution for elements of $\mathrm{C} 4$ cycle}

Although $S$ can be evaluated from equation (5.1) without knowing $b_{1}$ to $b_{4}$ explicitly, it is useful to have some way of testing whether or not the values found for $\alpha, \beta_{1}, \beta_{2}, \beta, \gamma$ and $\delta$ lead to a real solution for the $b$ 's. One approach to this is to use equations (3.19) and (3.20), which can be rewritten as

$$
\begin{aligned}
& \left(b_{2}+b_{4}\right) /\left(b_{1}+b_{3}\right)=a\left(b_{1}+b_{3}\right)^{2}-3 a b_{1} b_{3}+1-a, \\
& \left(b_{1}+b_{3}\right) /\left(b_{2}+b_{4}\right)=a\left(b_{2}+b_{4}\right)^{2}-3 a b_{2} b_{4}+1-a .
\end{aligned}
$$

This assumes that $\beta_{1}=\left(b_{1}+b_{3}\right)\left(b_{2}+b_{4}\right) \neq 0$, which means that we are excluding the solutions for which $\alpha=0$ (Section 2). These cases are easy to deal with separately.

If we assume $b_{1}+b_{3}=b_{2}+b_{4} \neq 0$, then equations (6.1) and (6.2) give $b_{1} b_{3}=$ $b_{2} b_{4}$ and as a result either $b_{1}=b_{2}$, with $b_{3}=b_{4}$ or $b_{1}=b_{4}$ with $b_{2}=b_{3}$. In either case we get an equilibrium solution. Thus we can deduce that for a $\mathrm{C} 4$ solution, with $\alpha \neq 0, b_{1}+b_{3}$ and $b_{2}+b_{4}$ are unequal. We shall assume that $b_{1}+b_{3}>b_{2}$ $+b_{4}$ for the rest of this section. Since $\alpha=\left(b_{1}+b_{3}\right)+\left(b_{2}+b_{4}\right)$ and $\beta_{1}=\left(b_{1}+\right.$ $\left.b_{3}\right)\left(b_{2}+b_{4}\right)$, we can obtain $b_{1}+b_{3}$ and $b_{2}+b_{4}$ as the roots of

$$
Z^{2}-\alpha Z+\beta_{1}=0 \text {. }
$$

If $\alpha^{2}<4 \beta_{1}$, this equation has complex roots and we can stop immediately. For unequal real roots we must have $\alpha^{2}>4 \beta_{1}$ and we can write

$$
b_{1}+b_{3}=(1 / 2)\left(\alpha+R_{1}\right), \quad b_{2}+b_{4}=(1 / 2)\left(\alpha-R_{1}\right),
$$

where $R_{1}=\sqrt{\left(\alpha^{2}-4 \beta_{1}\right)}$. This means that

$$
\begin{gathered}
\left(b_{1}+b_{3}\right)^{2}-\left(b_{2}+b_{4}\right)^{2}=\alpha R_{1}, \\
\left\{\left(b_{1}+b_{3}\right) /\left(b_{2}+b_{4}\right)\right\}-\left\{\left(b_{2}+b_{4}\right) /\left(b_{1}+b_{3}\right)\right\}=\alpha R_{1} / \beta_{1} .
\end{gathered}
$$

If we subtract equation (6.2) from (6.1) and use equations (6.5) and (6.6)

$$
3 a\left(b_{1} b_{3}-b_{2} b_{4}\right)=\left\{a+\left(1 / \beta_{1}\right)\right\} \alpha R_{1} .
$$

Taking $\alpha>0$, we get the result that

$$
b_{1} b_{3}>b_{2} b_{4} \text { provided } a+\left(1 / \beta_{1}\right)>0 \text {. }
$$

This latter condition held in all the cases that were considered, so we can take $b_{1} b_{3}$ as the larger root of the equation

$$
Z^{2}-\beta_{2} Z+\delta=Z^{2}-\left(b_{1} b_{3}+b_{2} b_{4}\right) Z+b_{1} b_{2} b_{3} b_{4}=0 .
$$


The roots of this equation must be real, since $b_{1} b_{3}+b_{2} b_{4}=\beta_{2}$ is real and $b_{1} b_{3}-b_{2} b_{4}$ is real, from equation (6.7). Hence we can write

$$
b_{1} b_{3}=(1 / 2)\left(\beta_{2}+R_{2}\right), b_{2} b_{4}=(1 / 2)\left(\beta_{2}-R_{2}\right) \text {, }
$$

where

$$
R_{2}=b_{1} b_{3}-b_{2} b_{4}=\sqrt{\left(\beta_{2}^{2}-4 \delta\right)} \text {. }
$$

From equation (6.7)

$$
R_{2}=\left(\alpha R_{1} / 3\right)\left\{1+\left(a \beta_{1}\right)^{-1}\right\} .
$$

We can now solve for $b_{1}$ and $b_{3}$, using $b_{1}+b_{3}=(1 / 2)\left(\alpha+R_{1}\right)$ and $b_{1} b_{3}=$ $(1 / 2)\left(\beta_{2}+R_{2}\right)$. If the roots are real and we take $b_{1}>b_{3}$, then

$$
b_{1}=(1 / 4)\left(\alpha+R_{1}+D_{1}\right), \quad b_{3}=(1 / 4)\left(\alpha+R_{1}-D_{1}\right),
$$

where

$$
\begin{aligned}
D_{1}^{2} & =\left(\alpha+R_{1}\right)^{2}-8\left(\beta_{2}+R_{2}\right) \\
& =2 \alpha^{2}-4 \beta_{1}-8 \beta_{2}-\left(2 \alpha R_{1} / 3\right)\left\{1+\left(4 / a \beta_{1}\right)\right\} .
\end{aligned}
$$

Unless $D_{1}^{2}>0$ the roots will not be real and distinct, since $D_{1}^{2}=0$ gives a C2 solution and $D_{1}^{2}<0$ gives complex values. In the same way, $b_{2}$ and $b_{4}$ are given by $(1 / 4)\left(\alpha-R_{1} \pm D_{2}\right)$, where

$$
D_{2}^{2}=2 \alpha^{2}-4 \beta_{1}-8 \beta_{2}+\left(2 \alpha R_{1} / 3\right)\left\{1+\left(4 / a \beta_{1}\right)\right\},
$$

provided $D_{2}^{2}>0$. Thus a sufficient condition for the solution to be real is that

$$
W=\operatorname{Min}\left(\alpha^{2}-4 \beta_{1}, D_{1}^{2}, D_{2}^{2}\right)>0 \text {. }
$$

Two minor notes are that $1+\left(4 / a \beta_{1}\right)$ is sometimes positive and sometimes negative, so $D_{2}^{2}-D_{1}^{2}$ is not always positive, and that the conditions $b_{1}+b_{3}>b_{2}$ $+b_{4}$, with $b_{1}>b_{3}$, do not imply that $b_{1}$ is the largest root. However, once the roots are known it is easy to arrange them in a suitable order.

\section{Numerical results}

The numerical work was carried out on a Univac 1100 computer, using double precision. An outline of the steps involved is given at the end of Section 4 and equations for $b_{1}, b_{2}, b_{3}, b_{4}$ are given in Section 6 . Only values of $a$ between 2 and 4 were used. The first step was to evaluate $M(Y, a)$ over a suitable range of values of $Y$ and $a$, to see how $M(Y, a)$ behaved and in particular to look for zeros. It was a surprise to find that $M(Y, 2)=0$ had a positive root and this root, which we 
shall call $Y_{4}$, could be followed throughout the interval $2 \leqslant a \leqslant 4$. Two additional roots appeared between $a=3.55$ and $a=3.6$ and a smaller root came in about 3.85. Finally, four larger roots appeared, in pairs, between $a=3.95$ and $a=4.0$. For $a=4$, all eight roots were recorded and they agreed with the values expected from the trigonometrical solutions. The occurrence of a single root for a polynomial of even degree, as recorded for $2 \leqslant a \leqslant 3.55$, looked a little bit strange but in fact it was accompanied by a negative root which we can label $Y_{1}$. For $a=2, Y_{1}$ was -2.68 and it increased smoothly with $a$, passing through zero around $a=3.85$ and being identified after that as a small positive root. We can think of $Y_{1}$ and $Y_{4}$ as a pair of roots which occur throughout the interval $[2,4]$ even although $Y_{1}$ is negative for part of this range and hence of no interest as far as real C4 solutions are concerned. Indeed it turns out that the $Y_{4}$ root does not always give real solutions either and it is this property which saves us from having C4 solutions for $a<1+\sqrt{5}$.

Apart from these families of roots, there were indications of an isolated zero at $a=2.535, Y=6.354$. Regarded as a function of two variables, $M(Y, a)$ has a minimum in this area and it appeared that the minimum was zero. This was checked more carefully and values of $M$ were obtained which were zero to 9 or 10 decimal places. The smallest value recorded on the computer print-outs was $-1 \times 10^{-11}$ for $a=2.535092575, Y=6.3542741$. As $Y^{8}$ equals $2.66 \times 10^{6}$ in this case, the fact that all the terms cancelled to an accuracy of $10^{-16} Y^{8}$ makes a good case for treating the minimum as zero. For these values of $a$ and $Y$, equations (4.10), (4.19) and (4.22) gave essentially the same linear relationship between $\beta_{1}$ and $\beta_{2}$, that is the ratios

$$
P_{1}: Q_{1}: R_{1}, P_{2}: Q_{2}: R_{2}, P_{3}: Q_{3}: R_{3},
$$

were the same to about eight decimal places. Because of this the usual method of determining $\beta_{1}$ and $\beta_{2}$ was ruled out. Instead, equation (4.10) was used to express $\beta_{2}$ as a linear function of $\beta_{1}$ and this expression was substituted for $\beta_{2}$ in equation (4.7), which gave a quadratic equation for $\beta_{1}$. The quadratic equation proved to have complex roots and this verified that the isolated zero did not lead to a real C4 solution.

Once the general pattern of the roots had been established, solutions were carried out for the $\mathrm{C} 4$ cycle corresponding to pairs $(Y, a)$ which gave $M(Y, a)=0$. The $Y$-values for $a=4$ were arranged in ascending order and labelled $Y_{0,1}, Y_{0,2}$, $Y_{1}, Y_{2}, \ldots, Y_{8}$, where $Y_{0,1}=Y_{0,2}=0$, and we can use these labels to identify corresponding families of roots. We can take $Y_{0,1}$ to refer to solutions with $\alpha=0$ and $2 a \beta=3(1-a)-Q$, while $Y_{0,2}$ refers to solutions with $\alpha=0$ and $2 a \beta=$ $3(1-a)+Q$. From Sections 2 and 5, the $Y_{0,1}$ solutions are stable for $a^{*}<a<$ $a^{* *}$, where the appropriate values for $a^{*}$ and $a^{* *}$ are listed in Table 1. The $Y_{0,2}$ solutions are unstable for $a>a^{*}$. 
TABLE 1. Critical values for solutions of period four and data for solutions with $a=4$.

\begin{tabular}{|c|c|c|c|c|c|c|}
\hline \multirow[b]{2}{*}{$\begin{array}{l}\text { Family of } \\
\text { solutions }\end{array}$} & \multirow[b]{2}{*}{$\begin{array}{l}a^{*} \\
a^{* *}\end{array}$} & \multirow[b]{2}{*}{$\begin{array}{l}\text { Stable } \\
\text { sequence }\end{array}$} & \multicolumn{4}{|c|}{ Data for solutions with $a=4$} \\
\hline & & & Root & $\begin{array}{l}\text { Numerical } \\
\text { Value }\end{array}$ & $\phi$ & $S$ \\
\hline$Y_{0,1}, Y_{0,2}$ & $\begin{array}{l}3.828427 \\
3.837665\end{array}$ & $Y_{0,1}$ & $\begin{array}{l}Y_{0,1} \\
Y_{0,2}\end{array}$ & $\begin{array}{l}0.0 \\
0.0\end{array}$ & $\begin{array}{l}4 \pi / 40 \\
5 \pi / 40\end{array}$ & $\begin{array}{l}+81 \\
+81\end{array}$ \\
\hline$Y_{1}$ & $\begin{array}{l}3.837665 \\
3.842116\end{array}$ & $Y_{1}$ & $Y_{1}$ & 0.119006 & $4 \pi / 41$ & -81 \\
\hline$Y_{2}, Y_{3}$ & $\begin{array}{l}3.547835 \\
3.548831\end{array}$ & $Y_{3}$ & $\begin{array}{l}Y_{2} \\
Y_{3}\end{array}$ & $\begin{array}{l}0.513167 \\
1.430098\end{array}$ & $\begin{array}{l}7 \pi / 40 \\
7 \pi / 41\end{array}$ & $\begin{array}{l}+81 \\
-81\end{array}$ \\
\hline$Y_{4}$ & $\begin{array}{l}3.236068 \\
3.288032\end{array}$ & $Y_{4}$ & $Y_{4}$ & 1.479355 & $8 \pi / 41$ & -81 \\
\hline$Y_{5}, Y_{6}$ & $\begin{array}{l}3.967535 \\
3.967556\end{array}$ & $Y_{5}$ & $\begin{array}{l}Y_{5} \\
Y_{6}\end{array}$ & $\begin{array}{l}9.379146 \\
10.0\end{array}$ & $\begin{array}{l}2 \pi / 41 \\
2 \pi / 40\end{array}$ & $\begin{array}{l}-81 \\
+81\end{array}$ \\
\hline$Y_{7}, Y_{8}$ & $\begin{array}{l}3.991929 \\
3.991938\end{array}$ & $Y_{8}$ & $\begin{array}{l}Y_{7} \\
Y_{8}\end{array}$ & $\begin{array}{l}19.486833 \\
20.512395\end{array}$ & $\begin{array}{l}\pi / 40 \\
\pi / 41\end{array}$ & $\begin{array}{l}+81 \\
-81\end{array}$ \\
\hline
\end{tabular}

For the $Y_{1}$ solutions, the critical value, $a^{*}$, occurs when $c_{8}=M(0, a)=0$. For $a<a^{*}, c_{8}$ is negative and $M(Y, a)$ has a negative root. For $a>a^{*}, c_{8}$ is positive and $Y_{1}$ takes positive values. It was noted that $a^{*}$ is slightly less than 3.85 and this suggests that the $Y_{1}$ solutions come in when the $Y_{0,1}$ solutions become unstable. For $a=3.84$, the $Y_{1}$ solution gave

$$
b_{1}=0.520350, \quad b_{2}=-0.936768, \quad b_{3}=-0.496237, \quad b_{4}=0.940069,
$$

and it will be seen that $b_{1}+b_{3}$ and $b_{2}+b_{4}$ are both close to zero. For the $Y_{0,1}$ solutions, $b_{1}+b_{3}$ and $b_{2}+b_{4}$ are exactly zero, so we can guess that the $Y_{1}$ solution and the $Y_{0,1}$ solution are similar. (For comparison, the $Y_{0,1}$ and $Y_{0.2}$ solutions for $a=3.84$ are $(0.511212,-0.938822,-0.511212,0.938822)$ and $(0.456836,-0.931304,-0.456836,0.931304)$. It will be seen that all three solutions are similar but the $Y_{1}$ solution is slightly closer to the $Y_{0,1}$ than to the $Y_{0,2}$ solution.) The numerical checks confirmed that $c_{8}=0$ when $a=3.837665$ and we can take this as the critical value for the $Y_{1}$ solutions. For this critical value the appropriate solution is the $Y_{0,1}$ solution and this gives $S=1$. As $a$ increases, $S$ decreases, passes through the value -1 and becomes -81 for $a=4$. The upper limit of $a$ for stability occurs when $S=-1$ and an appropriate value for $a$ was determined by interpolating from a table of values of $S$.

For the $Y_{4}$ family of roots, the corresponding $\mathrm{C} 4$ solutions are complex for $a<1+\sqrt{5}$. For $a>1+\sqrt{5}$ the solutions are real, with $S<1$. For $a=1+\sqrt{5}$ there is a C2 solution, with $b_{1}=1 / \sqrt{2}$ and $b_{2}=(1-\sqrt{5}) /(2 \sqrt{2})$ and with

$$
\left\{3 a b_{1}^{2}+(1-a)\right\}\left\{3 a b_{2}^{2}+(1-a)\right\}=-1 \text {. }
$$


If we think of this as the limiting case of a C4 solution, with $b_{3} \rightarrow b_{1}$ and $b_{4} \rightarrow b_{2}$ as $a \rightarrow 1+\sqrt{5}$ from above, then this limiting $C 4$ solution has $S=1$ and also $a \beta_{1}=-4,2 \alpha^{2}=4 \beta_{1}+8 \beta_{2}$. It follows that $D_{1}^{2}$ and $D_{2}^{2}$ are both zero (from equations (6.14) and (6.15)). For $a>1+\sqrt{5}$, the $C 4$ solutions are real, with $D_{1}^{2}$ and $D_{2}^{2}$ both positive. Something of this kind was to be anticipated, for equations (2.1) imply that $b_{2}$ and $b_{4}$ must be real when $b_{1}$ and $b_{3}$ are real. (Indeed as soon as one of the elements is real the others must be real.) For $a>1+\sqrt{5}, S$ decreases as $a$ increases and the limiting value of $a$ for stability occurs when $S=-1$.

The $Y_{2}$ and $Y_{3}$ roots have a maximum of $M$ between them (for a given value of $a)$ and the critical value, $a^{*}$, occurs when this maximum is zero and $Y_{2}, Y_{3}$ coincide. For $a \geqslant a^{*}$, the C4 solutions are real, with $S=1$ at $a=a^{*}$. As $a$ increases, $S$ decreases for the $Y_{3}$ family of solutions and there is a small interval within which the solutions are stable. For the $Y_{2}$ family of solutions, $S$ increases with $a$ and the $\mathrm{C} 4$ solutions are unstable for $a>a^{*}$.

For the $Y_{5}$ and $Y_{6}$ roots the pattern is similar. For a given value of $a$, there is a minimum of $M$ between them and the critical value occurs when the minimum is zero. For this critical value, $Y_{5}$ and $Y_{6}$ coincide and $S=1$. For larger values of $a$, $Y_{5}$ and $Y_{6}$ are distinct and correspond to real $\mathrm{C} 4$ solutions. For the $Y_{5}$ solutions there is a small interval of stability but the $Y_{6}$ solutions become unstable for $a>a^{*}$.

The $Y_{7}$ and $Y_{8}$ roots also come in as a pair, with a minimum of $M$ between the two roots. At the critical value of $a$, the two roots coincide and $S=1$. Each root leads to a real $\mathrm{C} 4$ solution. For the $Y_{8}$ solutions, $S$ decreases as $a$ increases and there is a small stability interval. For the $Y_{7}$ solutions, $S$ increases with $a$ and the solutions are unstable.

The appropriate values of $a^{*}$ and $a^{* *}$ are listed in Table 1 . It will be seen that the largest stability interval has a width of 0.052 , which gives a very sharp decrease in width compared with the largest interval for $\mathrm{C} 2$ solutions. The table also gives some information for the solutions which correspond to $a=4$. In particular, there is a column to indicate the range of values of $Y$ that is involved. The value of 10 for $Y_{6}$ is an exact result and the values for $Y_{2}$ and $Y_{7}$ are $10-3 \sqrt{10}$ and $10+3 \sqrt{10}$. This means that $Y-10$ and $Y^{2}-20 Y+10$ are factors of $M(Y, 4)$ and this was verified as a check on the coefficients in $M(Y, a)$.

\section{Acknowledgements}

The stimulus for this work came from Professor R. M. May's lecture notes for the Les Houches Summer School [7] and I am grateful to him for sending me a copy of these lecture notes. I should also like to thank Dr. S. M. A. Meggitt for 
his guidance on editing and control commands when compiling the computer programmes. The work has been done while appointed as a Visiting Fellow in the Research School of Physical Sciences, Australian National University, and I am grateful to the Department of Theoretical Physics for the facilities it has provided.

\section{References}

[1] M. Bôcher, Introduction to higher algebra (Macmillan, New York, 1907), 195-196.

[2] A. Brown, "Equations for periodic solutions of a logistic difference equation", J. Austral. Math. Soc. Ser. B 23 (1981), 78-94.

[3] A. Brown, "Solutions of period seven for a logistic difference equation", Bull. Austral. Math. Soc. 26 (1982), 263-284.

[4] A. Brown, "Solutions of period three for a non-linear difference equation", J. Austral. Math. Soc. Ser. B 25 (1984), 451-462.

[5] H. B. Griffiths, "Cayley's version of the resultant of two polynomials", Amer. Math. Monthly 88 (1981), 328-338.

[6] R. M. May, "Bifurcations and dynamic complexity in ecological systems", Ann. N. Y. Acad. Sci. 316 (1979), 517-529.

[7] R. M. May, "Non-linear problems in ecology and resource management", Lecture Notes for Les Houches Summer School on "Chaotic behaviour of deterministic systems", July 1981. 